\title{
Ultrasonographic Median Nerve Circumference, Thenar Muscle Thickness, and Grip Strength Change and Correlation After Carpal Tunnel Release: Single Case Design
}

\section{Tony Boucher*}

Department of Health and Kinesiology, Texas A\&M University, United States

*Corresponding Author: Tony Boucher, Department of Health and Kinesiology, Texas A\&M University, United States.
Received: October 18, 2021

Published: November 03, 2021

(C) All rights are reserved by Tony Boucher.

\section{Abstract}

Purpose: The purpose of this study was to assess change and correlation of median nerve circumference, abductor pollicis brevis muscle thickness, and hand grip strength post carpal tunnel release.

Subject: A 47-year-old woman post open carpal tunnel release was immobilized in a neutral wrist splint for six weeks then began a progressive two-month rehabilitation program supervised by a physical therapist.

Methods: Both transverse median nerve circumference and transverse abductor pollicis brevis muscle thickness were assessed using rehabilitative ultrasound imaging and grip strength was measured utilizing a hand-held dynamometer preoperatively and every four weeks postoperatively for nine months.

Data analysis: Repeated measures analysis of variance evaluated median nerve circumference, abductor pollicis brevis muscle thickness, and grip while Pearson correlation coefficient measured their relationships.

Results: Median nerve circumference showed significant reduction preoperative to month 9 ( $1 \mathrm{~mm}$ to $0.64 \mathrm{~mm}, \mathrm{p}=0.018$ ), month 2 to 5 (1.02 mm to $0.92 \mathrm{~mm}$; $\mathrm{p}=0.034)$, and month 3 to $6(1.1 \mathrm{~mm}$ to $0.92 \mathrm{~mm}$; $=0.037)$. Abductor pollicis brevis muscle thickness significantly increased every month $(1.29 \mathrm{~cm}$ to $1.43 \mathrm{~cm}, \mathrm{p}=0.001)$ except month 1 and 4 . Grip significantly decreased preoperative to month 1 (20.87 $\mathrm{kg}$ to $16.33 \mathrm{~kg}, \mathrm{p}=0.044$ ) but significantly increased through month 8 (20.87 $\mathrm{kg}$ to $25.40 \mathrm{~kg}$, p = 0.031). Large immediate postoperative median nerve largely correlated with low grip strength $(r(3)=-0.866)$. At month 8 , small median nerve largely correlated with high grip strength $(r(3)=-0.5)$ and the large correlation between small median nerve and large abductor pollicis brevis muscle thickness was significant $(r(3)=-0.952, p=0.012)$.

Conclusions: Slightly increased postoperative median nerve circumference and healing influenced grip and abductor pollicis brevis muscle thickness initially but progressively improved over nine months. Gradual muscle muscle thickness hypertrophy and recovery of grip strength post carpal tunnel release appears directly related to progressive decrease median nerve circumference.

Clinical Relevance: The patient returned to full activity and work function at 8 weeks post carpal tunnel release but continued to exhibit strength and thenar muscle deficits that correlated to postoperative median nerve inflammation and recovery that persisted into the eighth month.

Keywords: Carpal Tunnel Release; Median Nerve; Thenar Muscle Thickness; Grip Strength; Ultrasound Imaging

Citation: Tony Boucher. "Ultrasonographic Median Nerve Circumference, Thenar Muscle Thickness, and Grip Strength Change and Correlation After Carpal Tunnel Release: Single Case Design". Acta Scientific Orthopaedics 4.12 (2021): 04-08. 


\section{Abbreviations}

APB: Abductor Pollicis Brevis; CTR: Carpal Tunnel Release; CTS: Carpal Tunnel Syndrome; MT: Muscle Thickness; MN: Median Nerve; RTUS: Real Time Ultrasound Imaging; s/p-Status Post

\section{Introduction}

The carpal tunnel is a fibro-osseous complex through which the anterior muscles pass through the wrist. The floor is formed by the proximal carpal bones and the roof by the transverse carpal ligament (flexor retinaculum). The flexor pollicis longus tendon, four slips of the flexor digitorum superficialis, four slips of the flexor digitorum profundus and the median nerve (MN) also occupy this tunnel. Inflammation of the structures inherent to the tunnel and factors that decrease the space in the tunnel may compress or restrict the mobility of the median nerve creating carpal tunnel syndrome (CTS). This causes a compression or traction nerve injury with neurological symptoms in the distal median nerve distribution, weakness, decreased grip and pinch strength, and atrophy of the thenar muscles supplied by the median nerve. The abductor pollicis brevis (APB) and opponens pollicis are two of the primary thumb muscles supplied by the median nerve and compromised as a result of CTS.

Compression of the median nerve at the carpal tunnel has been shown to produce morphological swelling and inflammation of the nerve compared to unaffected nerves. Patients with CTS have greater transverse, anteroposterior, and cross sectional area dimensions compared to controls [1,2]. Surgical intervention and management is often required if conservative measures do not relieve the symptoms. Surgical decompression involving the transection of the transverse carpal ligament is performed to increase the volume of the carpal tunnel and relieve compression on the median nerve. Surgical options are open carpal tunnel release (CTR) or endoscopic assisted carpal tunnel release.

Rehabilitative or real time ultrasound imaging (RTUS) is an emerging technology in rehabilitative medicine to assess nerve in response to pathology and trauma, and to measure skeletal muscle size. Physiologically and anatomically, nerve size is correlated to inflammation and muscle size is correlated to muscle function. Measurements of nerve and muscle size provide indirect evidence of functional recovery. Objective measurements to quantify the out- come of surgical management and progression with rehabilitation of CTR are needed to quantify recovery $[5,6]$. RTUS has proved to be an excellent imaging method to demonstrate median nerve entrapment in the carpal tunnel. Previous studies have shown RTUS to be fast, efficient, reliable method with excellent specificity and sensitivity for median nerve morphology associated with CTS [14]. In addition, a decrease in the cross-sectional area of the median nerve after the release of the transverse carpal ligament has also been shown and the cross-sectional area of the median nerve was the only predictor of success after three months post CTR in patients with idiopathic CTS $[5,6]$. A combination of objective findings that include RTUS also improves the prediction after CTR [6]. Multiple studies, including systematic reviews, have concluded that RTUS is a reliable and valid objective measure of muscle thickness, cross-sectional area, and muscle volume in varying populations and conditions [7-19]. RTUS is also cost-effective, non-invasive, and safe. The purpose of this single case design clinical study was to investigate the recovery of the median nerve and thenar muscle morphological changes using RTUS in correlation to thenar muscle strength post CTR.

\section{Case Presentation}

A forty-seven year old healthy female post open carpal tunnel release was immobilized in a neutral wrist splint for 6 weeks then began a progressive 2-month rehabilitation program supervised by a licensed physical therapist. Transverse median nerve circumference (Figure 1) and transverse abductor pollicis brevis muscle thickness (MT) (Figure 2) using rehabilitative ultrasound imaging (Figures 3 and 4) and hand grip utilizing a hand-held dynamometer were assessed preoperatively and every 4 weeks for 9 consecutive months. Repeated measures analysis of variance examined MN, APB, and grip while Pearson correlation coefficient analyzed their relationships. MN circumference showed significant reduction preoperative to month 9 ( $1 \mathrm{~mm}$ to $0.64 \mathrm{~mm}, \mathrm{p}=0.018$ ), month 2 to 5 (1.02 $\mathrm{mm}$ to $0.92 \mathrm{~mm} ; \mathrm{p}=0.034)$, and month 3 to 6 (1.1 mm to $0.92 \mathrm{~mm} ; \mathrm{p}=0.037)$. APB MT significantly increased every month (1.29 $\mathrm{cm}$ to $1.43 \mathrm{~cm}, \mathrm{p}=0.001)$ except 1 and 4 . Grip significantly decreased preoperative to month 1 (20.87 $\mathrm{kg}$ to $16.33 \mathrm{~kg}, \mathrm{p}=0.044$ ) but significantly increased through month 8 (20.87 $\mathrm{kg}$ to $25.40 \mathrm{~kg}, \mathrm{p}=0.031$ ). Large immediate postoperative MN circumference largely correlated with low grip strength $(\mathrm{r}(3)=$ -0.866). At month 8, small MN circumference largely correlated 
with high grip strength $(\mathrm{r}(3)=-0.5)$ as well as a large correlation between small MN circumference and greater APB MT was significant $(r(3)=-0.952, p=0.012)$.

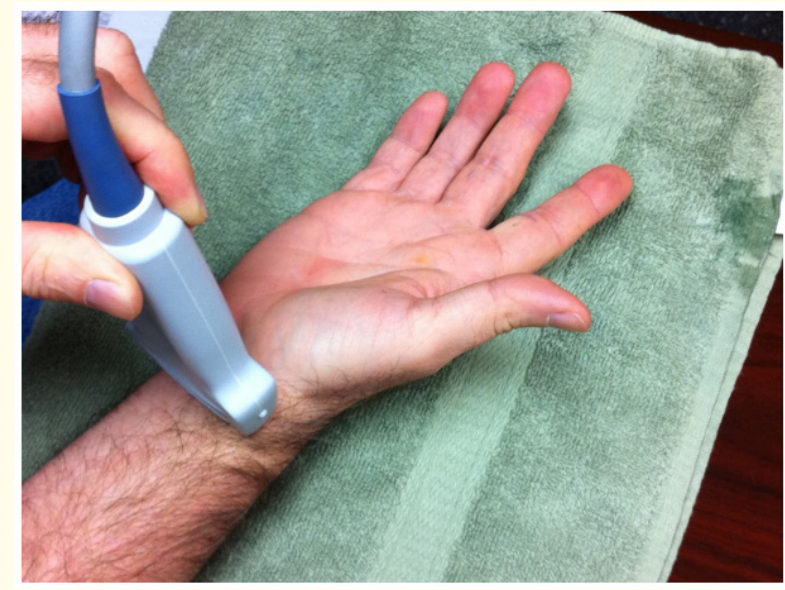

Figure 1: Example of rehabilitative ultrasound imaging after carpal tunnel release of median nerve at the carpal tunnel from the Sonosite M-Turbo (Sonosite, Inc., Bothell, WA).

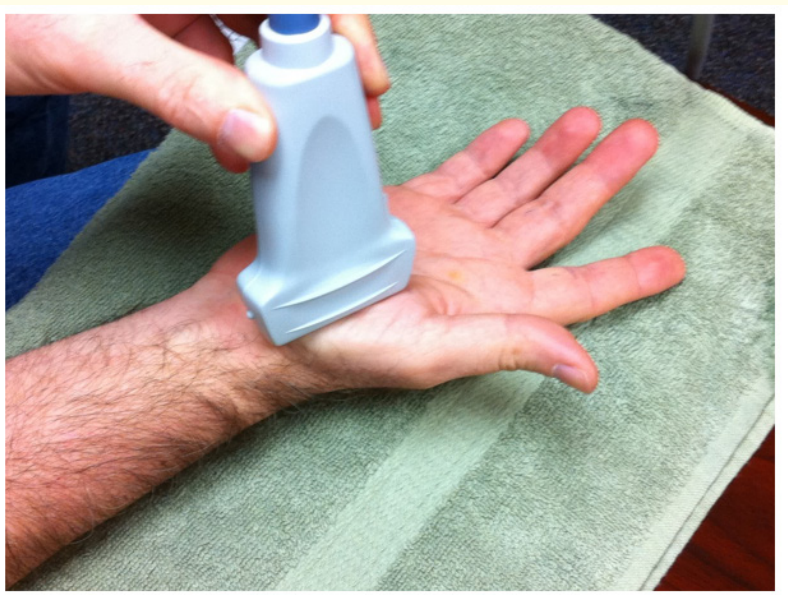

Figure 2: Example of rehabilitative ultrasound imaging after carpal tunnel release of abductor pollicis brevis muscle from the Sonosite M-Turbo (Sonosite, Inc., Bothell, WA).

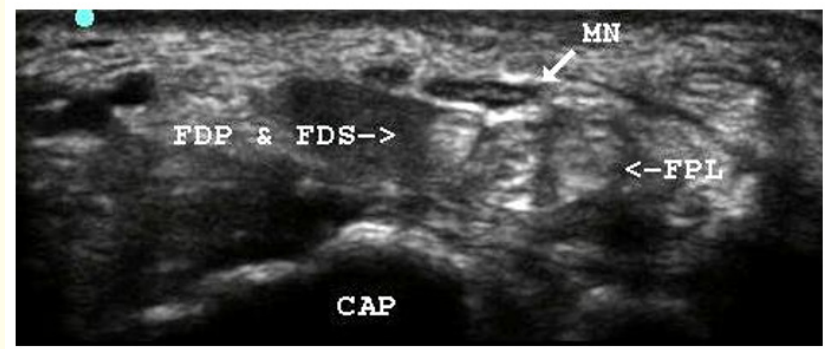

Figure 3: Sample transverse image of the median nerve at the carpal tunnel from the Sonosite M-Turbo (Sonosite, Inc.,

Bothell, WA) using a 13-6 MHz multi-frequency, broadband, 25 $\mathrm{mm}$ linear array in nerve optimized B mode at $1.5 \mathrm{~cm}$ depth. Abbreviations: MN, median nerve; FPL, flexor pollicis longus tendon; FDP, flexor digitorum profundus tendons; FDS, flexor digitorum superfiscialis tendons; CAP, capitate bone.

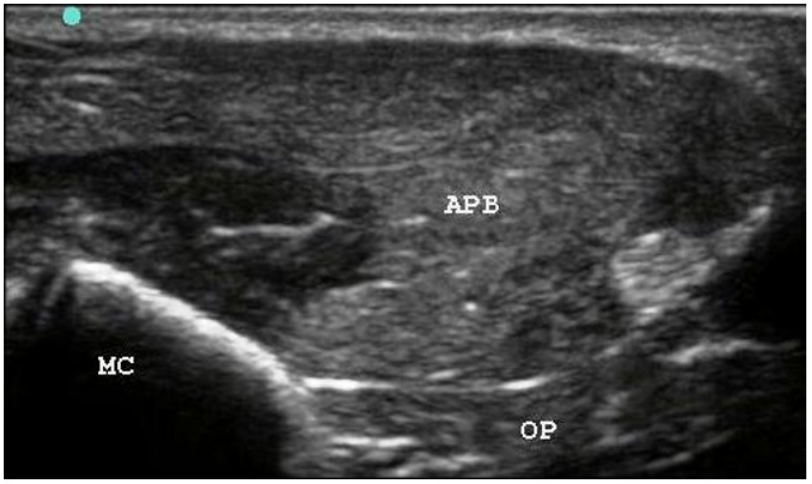

Figure 4: Sample transverse image of the abductor pollicis brevis muscle from the Sonosite M-Turbo (Sonosite, Inc., Bothell, WA) using a 13-6 MHz multi-frequency, broadband, $25 \mathrm{~mm}$ linear array in musculoskeletal optimized B mode at $2.2 \mathrm{~cm}$ depth. Abbreviations: ABP, abductor pollicis brevis; OP, opponens pollicis; MC, $1^{\text {st }}$ metacarpal.

\section{Discussion}

Carpel tunnel syndrome is a common orthopedic pathology afflicting numerous people. The condition is most often chronic 
with neural pressure and inflammation that creates atrophy of hand musculature, decreased grip, and altered hand function $[2,3,6]$. Decompression of the medial nerve through a carpel tunnel release procedure is often the chosen resolution. While acute symptoms and sensation often improve, patients recover in varying capacities post-surgery [5,6]. Initial strength gains are due to neural adaptation and are postulated to be due to an increase in recruitment as well as increased rate and synchronization of firing. Later gains in muscle function are due to hypertrophy and hyperplasia of muscle fibers.

With carpal tunnel release, the decompressed MN is no longer restricted by the anatomical confines of the carpal tunnel [6]. Generalized post-operative inflammation of the wrist complex is expected post decompression and while the MN is unconstrained, the neural healing process is time dependent [5]. Once postoperative MN inflammation improves, the neuromuscular efficiently, recruitment, and strength gains progress temporally.

Post-operative CTR resolution is evident by assessing the medial nerve circumference as a relative indicator of inflammation, muscle thickness as an indicator of hypertrophy, and grip strength as an indicator of function [2]. This case displayed slightly increased postoperative median nerve circumference with subsequent healing that influenced grip and thenar muscle thickness initially but progressively improved over nine months in direct correlation to decreasing MN size.

\section{Conclusion}

Gradual muscle hypertrophy and recovery of grip strength post carpal tunnel release appears directly related to progressive decrease median nerve inflammation and circumference. The reported case returned to full activity and work function at eight weeks post-operative but continued to exhibit strength gains over time that correlated to median nerve recovery and decreasing postoperative inflammation that persisted into the eighth month but with continued correlated improvement.

\section{Acknowledgements}

None.

\section{Conflict of Interest}

No conflict of interest.

\section{Bibliography}

1. Duncan I., et al. "Sonography in the diagnosis of carpal tunnel syndrome". American Journal of Roentgenology 173.3 (1999): 681-684.

2. Wiesler ER., et al. "The use of diagnostic ultrasound in carpal tunnel syndrome". The Journal of Hand Surgery 31.5 (2006): 726-732.

3. Buchberger W., et al. "Carpal tunnel syndrome: Diagnosis with high-resolution sonography". American Journal of Roentgenology 159.4 (1992): 793-798.

4. Wong SM., et al. "Carpal tunnel syndrome: Diagnostic usefulness of sonography”. Radiology 232.1 (2004): 93-99.

5. Abicalaf CA., et al. "Ultrasound evaluation of patients with carpal tunnel syndrome before and after endoscopic release of the transverse carpal ligament". Clinical Radiology 62.9 (2007) 891-894.

6. Naranjo A., et al. "Usefulness of clinical findings, nerve conduction studies and ultrasonography to predict response to surgical release in idiopathic carpal tunnel syndrome". Clinical and Experimental Rheumatology 27.5 (2009): 786-793.

7. Audenaert EA., et al. "Deltoid muscle volume estimated from ultrasonography: In vitro validation and correlation with isokinetic abduction strength of the shoulder". Medical and Biological Engineering and Computing 47.5 (2009): 557-563.

8. Bemben MG. "Use of diagnostic ultrasound for assessing muscle size". Journal of Strength and Conditioning Research 16.1 (2002): 103-108.

9. Emshoff R., et al. "Reliability and temporal variation of masseter muscle thickness measurements utilizing ultrasonography”. Journal of Oral Rehabilitation 30.12 (2003): 1168-1172.

10. Hides JA., et al. "Magnetic resonance imaging and ultrasonography of the lumbar multifidus muscle. comparison of two different modalities". Spine 20.1 (1995): 54-58. 
11. Hides JA., et al. "Diagnostic ultrasound imaging for measurement of the lumbar multifidus muscle in normal young adults". Physiotherapy Theory and Practice 8.1 (1992): 19-26.

12. Kazuto W., et al. "Use of ultrasonography to evaluate thickness of the erector spinae muscle in maximum flexion and extension of the lumbar spine". Spine 29.13 (2004): 1472-1477.

13. Kiesel KB., et al. "Measurement of lumbar multifidus muscle contraction with rehabilitative ultrasound imaging". Manual Therapy 12.2 (2007): 161-166.

14. McMeeken JM., et al. "The relationship between EMG and change in thickness of transversus abdominis". Clinical Biomechanics 19.4, (2004): 337.

15. Miyatani M., et al. "Validity of ultrasonograph muscle thickness measurements for estimating muscle volume of knee extensors in humans." European Journal of Applied Physiology 86.3 (2002): 203-208.

16. Miyatani M., et al. "The accuracy of volume estimates using ultrasound muscle thickness measurements in different muscle groups". European Journal of Applied Physiology 91.2 (2004): 264-272.

17. Pretorius A and Keating JL. "Validity of real time ultrasound for measuring skeletal muscle size". Physical Therapy Reviews 13.6 (2008): 415-426.

18. Reeves ND., et al. "Ultrasonographic assessment of human skeletal muscle size". European Journal of Applied Physiology 91.1 (2004): 116-118.

19. Wallwork TL., et al. "Intrarater and interrater reliability of assessment of lumbar multifidus muscle thickness using rehabilitative ultrasound imaging". Journal of Orthopaedic and Sports Physical Therapy 37.10 (2007): 608-612.

\section{Volume 4 Issue 12 December 2021}

(C) All rights are reserved by Tony Boucher.

Citation: Tony Boucher. "Ultrasonographic Median Nerve Circumference, Thenar Muscle Thickness, and Grip Strength Change and Correlation After Carpal Tunnel Release: Single Case Design". Acta Scientific Orthopaedics 4.12 (2021): 04-08. 